\title{
EVALUATING PAAS SCALABILITY AND IMPROVING PERFORMANCE USING SCALABILITY IMPROVEMENT SYSTEMS
}

\author{
Nishant Agnihotri', Aman Kumar Sharma ${ }^{2}$ \\ ${ }^{I}$ Assistant Professor, Department of Computer Science \& Application, Indus International University, Himachal Pradesh, \\ India \\ ${ }^{2}$ Associate Professor, Department of Computer Science, Himachal Pradesh University, Himachal Pradesh, India
}

\begin{abstract}
Cloud computing has almost changed the way of obtaining resources and managing platform as a service. With these improvements, challenges like scalability testing, performance testing is emerging very fast. This paper focuses on the evaluation of scalability of PaaS as well along with that propose a graphical model where SIS (Scalability Improvement System)is proposed in which cache are implemented at the both interfaces of PaaS to enhance the scalability in terms of providing service to the user.
\end{abstract}

Keywords: Evaluating PaaS scalability, scalability testing, proposed graphical model of SIS.

\section{INTRODUCTION}

Starting with the evolution in the field of technology the generations comes first in mind. Cloud computing $[8,1,5]$ is the result of these advancements in the technologies or the generation in the field of technology. In the very first stage when there were large computers and the costs were very high the results were not satisfactory but were costly. With the enhancement in the technology the industry/technology moved toward the IC's and the computation time was reduced to a large extent with the enhancement in the storage capacity. After these stages the next stage was the "cloud computing" where the computation cost and storage were given the main importance. Cloud computing is the result of combination of various services which are clubbed together to give a massive change to the new era.

Many technologies clubbed together to give result in form of cloud computing. These services are grid computing, hybrid computing, parallel computing, network storage technology and many other technologies. In short cloud computing gave birth to the on demand service with the help of some laid infrastructure. User can demand for any service it can be software, storage, or any other internet service.

In order to implement cloud computing [8] a basic thing required is the Infrastructure/Architecture which is combination of various services.

- IaaS (Infrastructure as a Service): the very first component/layer of the architecture where the user gets a platform of a computer system with the help of internet.

- PaaS (Platform as a Service): the second component/layer of the architecture where a platform for the development of the software is given to the user though internet.
- SaaS (Software as a Service): the third component/layer of the architecture where a user can use any software as per the need through the internet.

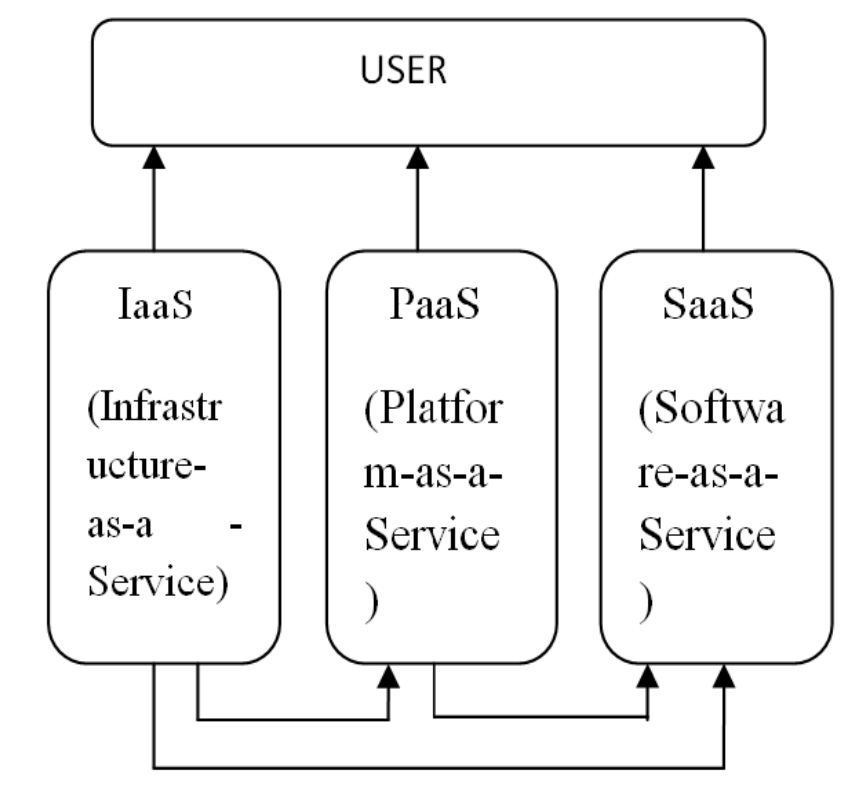

Fig1: Cloud computing infrastructure /architecture

Most of the research works in the last few years have focused toward the performance evaluation and scalability analysis. Hence, this research paper has a purpose to support evaluation of performance and scalability analysis for cloud based applications provides platform for development such as PaS (Platform as a Service), SaaS (Software as a Service) and IaaS 
(Infrastructure as a Service) because of some distinguished features.

- Providing the scalability to the applications based on the cloud is the major advantage and a distinguished feature. Assuring this claims the basic and the important issue, and can be assured by the evaluation of performance parameters.

- All SaaS (Software as a Service) in cloud are operated with the physical and virtual computing resources with the support of scalable infrastructure.

- Using PaaS (Platform as a Service), with the use of efficient virtualized computing resources that can be scaled up and down rapidly in a secure way to deliver a high quality of service new applications can made available [12]

\subsection{Research Objective}

- The objectives of the research paper are formulated as under:

- Identifying the scalability factors on PaaS.

- Analyzing the various problems scaling down the Scalability.

- Improve the efficiency of cloud network with the implementation of Scalability Improvement System.

\subsection{Literature Review}

Scalability testing $[11,12,4]$ and performance evaluation in traditional and cloud based applications

There are four primary objectives in evaluating PaaS for the scalability.

- Check whether the resources are properly allocated to ensure delivery of service to end user.

- Identify whether the performance parameters are analyzed properly in order to ensure the connectivity.

- Properly analyze the performance of end system in term of accessing resources.

- Properly analyze the scalability PaaS at the cloud in order to assure the quality of service.

There is a big gap and difference in evaluating the scalability or performance of a traditional and cloud based application [8]. There are various factors which are helpful in order to analyze those gaps as the major concern in testing the cloud based application [7] is fully based on the scalability as well as the performance in term of delivery of service.

Whereas in traditional software [13] there are some more factors which are bit different than cloud. Some more factors are discussed below

- All the applications which works on the cloud environment provide a contract to the customers known as the SLA [6] (Service Level Agreement) which focuses on the quality of service, as the quality is the major part of the cloud computing in that case this agreement is thoroughly verified and validated by the Provider of SaaS, PaaS and IaaS.

- Cost of using data by customer should not more than the service used.

- Testing in cloud need not to buy any specific software for the testing whereas in traditional testing this becomes an unaffordable cost as cloud provides an infrastructure that is capable of performing testing on various software on single platform.

- It becomes difficult to create test environments as the traditional software depends upon various parameters and configurations as compared to the cloud. These parameters are, enterprise level firewall configuration, network configuration and registry settings. Combination of these parameters grows by the geometry [8], but the resultant configuration is difficult to manage.

Testing itself playing a common role in both the traditional testing but still there are few factors which came out when testing is done in cloud as the variety of testing plans and Schemes are provided by the provider of the service. Benefits of cloud testing [8] over traditional testing are as follows

- Cloud incurs financial difficulty: it is an economical aspect of cloud computing where user pays as per the use i.e. the amount of data used the proportional will be the charge [10]. This overcomes the financial difficulty from the consumer point of view.

- Cloud saves the jitter: it is an effectiveness improving aspect of cloud computing where the services are assured to be the fastest one. Delays are minimized due to implementation of new technologies as it is fully network driven approach so it improves the packet transfer speed.

- Clouds are easy to expand: it is a fully manageable network driven approach which ensures scalability. It is easy to expand by adding new devices to the network resulting into an easily manageable and expandable network.

- Cloud respond rapidly: In traditional testing where testing environment is created for different software and different test cases are designed. Cloud testing saves the time money and respond time by providing virtualized environment to the consumer. For the implementation of the testing with the number of test cases as much work is finished earlier like implementation of hardware and software installation, environment maintenance, middleware configuration. And because of the existing environment respond is given immediately.

- PaaS scalability: Platform as a Service acts as an intermediate layer in the cloud computing our idea approaches toward the various factors for the scaling up as well as scaling down various components in PaaS. These factors are mentioned below.

- Factors considered to scale up: evaluating the platform for various developments, whether it provides the compatible platform for the rising demand of new technologies. 
- Factors consider to scale down: identifying the various factors responsible for the scaling down the scalability even after the load is been removed from the network.

- Cost for the scalability: identifying the various cost factors for the utilization of resources responsible for the scalability improvement.

\section{PROPOSED MODEL FOR THE SCALABILITY IMPROVEMENT}

This section explains the brief description about the cost factor in PaaS where economical aspect of cloud computing where user pays as per the use is considered primarily. A mode is proposed(Figure 2) based on the old networks where up gradation with the new devices will be much costly rather than cutting down the cost of up gradation with in implementation of a cache scheme on the PaaS layer. As discussed by C.Baker [2] in his research about the enhancing the scalability by adding new manageable switches to the network where some new devices were added to the network. In order to scale down the cost of the use and improving scalability this step was a successful attempt. But on the other hand the cost of replacement was still a major issue. Whereas T.Banditwattanawong [11] proposed a model where a cache on the client-side is implemented which was a successful attempt to improve the scalability and reduction of cost in terms of SaaS(Software as a Service), but on the cloud side the scalability in terms of PaaS and IaaS was still a major issue.

SIS (Scalability Improvement System): SIS is a combination of Load balancer, Load analyzer and caches. Load analyzer; analyze all the requests which are made by the user to the system directly or via SaaS. It will keep a track of repeatedly requested data and saves repeatedly requested elements on the cache 1 or cache 2 depending upon the interface whether connected to SaaS or IaaS. When the same item is requested next time it will be provided effectively by the respective cache. On the other hand when any of the cache overflows, Load balancing mode will get activated and transfer the overloaded data to the other cache resulting in the fast access to the repeatedly requested elements. Figure (2) shows the proposed system and its implementation with the architecture of Cloud computing. Whereas Figure (3) represents the internal components of the SIS (Scalability Improvement System) is responsible for the effective functioning of the system.

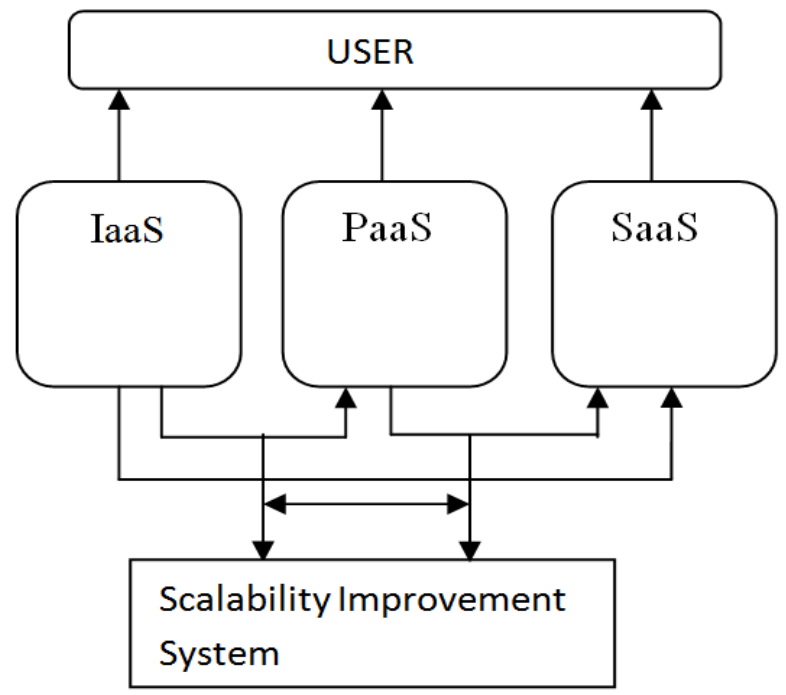

Fig 2: Proposed Scalability Improvement system

\begin{tabular}{|l|l|l|}
\hline Cache 1 & Load analyzer & Cache 2 \\
\hline \multicolumn{3}{|c|}{ Scalability Improvement system } \\
\hline
\end{tabular}

Fig3: Components of Proposed Scalability Improvement system

- Cache 1: Cache dedicatedly works for the interface connected to the IaaS and PaaS. Keep the small objects with the higher demand from the customer in order to save the respond time of repeatedly occurring or demanding item. Cache 1 can work as a load balancing cache to cache 2.

- Cache 2: Cache dedicatedly works for the interface connected to the SaaS and PaaS. Keep the small objects with the higher demand from the customer in order to save the respond time of repeatedly occurring or demanding item. Cache 2 can work as a load balancing cache to cache 1.

- Load analyzer: The major component of SIS with a control strategy which will decide the activation of Load Balancing mode depending upon the size of data on each cache.

- Load Balancing Mode: Mode of SIS where when any of cache have load more than other in order to improve the efficiency this mode will enable and transfer the further load to the other cache. 


\subsection{Functionality \& Advantages of Proposed System}

SIS works on following steps

1. Assign more contents to the cache which is freer or not in use: Set a weight on the scale for each cache and the data will be proportionally distributed on the cache.

2. Route traffic to the dedicated cache till it is available: by arranging the distribution priority order of data transfer, the dedicated cache will get the data on first priority. Lower priority cache will only be used if the current cache gets overloaded.

3. Give the fastest response time when using applications like Software Development Kit and more online development tools.

\section{SIS(C1,C2,LA,REQ)}

$\mathrm{C} 1$ : cache 1

$\mathrm{C} 2$ : cache 2

LA: Load analyzer (Analyze the load on

cache $1 \&$ cache 2 )

REQ: Request (item) (Request for the item by the client)

Step 1: START

Step 2: LA $\leftarrow$ REQ(ITEM)

Step 3: IF REQ(ITEM) $=$ REQ $($ IaaS $) \& \& I T E M==L A(I T E M)$

$\& \&$ size $(\mathrm{C} 1)>\mathrm{REQ}(\mathrm{ITEM})$

Step $4:$ LA=REQ(ITEM), C1

Step 5: ELSE IF REQ(ITEM) $=\operatorname{REQ}(\operatorname{SaaS}) \& \&$ ITEM== LA(ITEM)\&\& size(C2)> REQ(ITEM),

Step 6: C2 -REQ(ITEM)

Step 7:ELSE ACTIVE LB.

Step 8: STOP

Steps followed by Load balancing

LB: load balancer (balances the load of the cache when load balancing mode is active)

$\mathrm{LB}(\mathrm{C} 1, \mathrm{C} 2)$ (load balancer, gets active when cache 1 or cache 2 overflows)

Step 1: ANALYZE C1\&C2

Step 2: IF SIZE(C1)>SIZE(C2)

Step 3: TRANSFER REQ(ITEM) $\rightarrow \mathrm{C} 2$

Step 4: ELSE TRANSFER REQ(ITEM) $\rightarrow \mathrm{C} 1$

Step 5: STOP

\section{Advantages of Scalability Improvement System:}

- Scaling down delays: After implementation of the cache on the layers responding to the repeatedly demanded data will be improved. Load balancing scheme provides the backup to the cache resulting in the improvement of the scalability. As a result the delays will get scaled down.

- Scaling down the usages cost: As a result of improved scalability and the fast responding property, used needs not to wait for the elements for a long time, with this the time is saved which also improves the cost factor. As a result the usage cost scaled down up to a good extent.

- Scaling up the connectivity: After implementing SIS (Scalability Improvement System) connectivity between the layers become double, requests waiting time will get scale down up to a good extent, which results in good connectivity.

- Scaling down the implementation cost: In case of improving the scalability the preference are given to change of topology, replacing switches or various servers which scales up the cost of implementation. Whereas the proposed SIS can work upon any pre implemented topology and devices which

\section{CONCLUSIONS}

With the emergence of new technologies on the cloud testing and scalability have become a tedious job. There are number of other methods which work for the testing purpose of application/web applications/development testing the scalability and for its improvement. The latest/emerging techniques offer new setup rather than improvement of the traditional setups/devices. Although scalability testing and its improvement is the major issue but replacement of traditional systems is not the solution at all. SIS is useful for the traditional/current setups on the cloud. SIS enhances the scalability along with the reduction in the cost of use of the network. Future work involves controlling of overflow of the requests by the customer for multiple items at a single time if occurs in the Scalability Improvement System and the implementation of the testing on Scalability Improvement System.

\section{REFERENCES}

[1]. Atif Farid Mohammad and Hamid Mcheick, "Cloud Service Testing: An Understanding", The 2nd International Conference on Ambient Systems, Network and Technologies, ELSEVIER, 2012, pp 513-520.

[2]. C.Baker, A.Anjum, R.hill, N.bessis and S.L.Kiani,"'Improving cloud datacenter scalability, Agility and performance using open flow", IEEE 4th International Conferences on Intelligent Networking and Collaborative Systems, 2012, pp 20-27

[3]. D.Chappell,"A Short introduction to Cloud Platform" David Chappel and Associates, 2008

[4]. D.Jayasinghe, S.Malkowski, J.li, Q.Wang, Z.Wang, And Calton $\mathrm{Pu}$, "Variation In Performance and Scalability: An Experimental Study in IaaS Clouds using Multi-Tier Workloads", IEEE TRANSACTIONS ON SERVICE COMPUTING, IEEE, 2013, pp 1-14.

[5]. G.Gruman" What Cloud Computing really Means", InfoWorld, 2009

[6]. J.Gao, P.Pattabhiraman, X. Bai, W.T.Tsai, "SaaS Performance and Scalability Evaluation in Clouds", 6th International Symposium on Service Oriented System Engineering, IEEE, 2011, pp 61-71.

[7]. M.A.vouk, "Cloud Computing-issues, Research and Implementation [J]", Journal of Computing and Information Technology, CIT 16, 2008 (4), pp 235-246. 
[8]. Peng Zhelonlong, Ou Yang Zhonghuli and Huang Youlan, "The Application and Development Of Software Testing in Cloud Computing Environment", IEEE International conference on Computing Service System, 2012, pp 450-454.

[9]. R.Buyya, Y.S.Chee and V. Srikumar,"Market-Oriented Cloud Computing: Vision, hype and Reality for Delivering IT Services as Computing Utilities", Department of Computer Science and Software Engineering, University of Melbourne Australia, 2008, pp 9.

[10]. T. Banditwattanawong,"From Web Cache to Cloud Cache", 7th International Conf In Grid and Pervasive Computing, Hong-Kong, 2012, pp 1-15

[11]. Thepparit Banditwattanawong and Putchong Uthayopas, "Improving cloud scalability, economy and responsiveness with client-side cloud cache" IEEE, 2013.

[12]. Trieu C. Chieu, Ajay Mohindra and Alexei A. karve, "Scalability and Performance of Web Application in a Computing Cloud", 8th International Conference on ebusiness Engineering, IEEE 2011, pp 317-323.

[13]. Zhao Bin, "Use of Senior Software Test Engineerclassic Tutorial of Software Testing Technology", [M] Science Press, 2007, pp- 29-46. 